\title{
On the Effectiveness of the 2-hop Routing Strategy in Mobile Ad Hoc Networks
}

\author{
Michele Garetto $^{\dagger}$ Paolo Giaccone* Emilio Leonardi * \\ * Dipartimento di Elettronica, Politecnico di Torino, Italy \\ $\dagger$ Dipartimento di Informatica, Università di Torino, Italy
}

\begin{abstract}
In this paper, we study the performance of the 2-hop routing scheme proposed for ad hoc wireless networks with mobile nodes, considering realistic node mobility patterns. First, we provide a formal definition of optimal routing maximizing the throughput of a mobile ad hoc network, in terms of a multi-commodity flow problem over the associated contact graph. Then, we relate the effectiveness of the 2-hop routing strategy to structural properties of the contact graph. We present experimental results showing that, in real networks, contact times among the nodes are largely inhomogeneous. Our results show that, in networks with inhomogeneous contact times, the 2-hop routing strategy can result strongly inefficient in terms of network throughput.
\end{abstract}

\section{INTRODUCTION AND PREVIOUS WORK}

In their seminal work [1], Grossglauser-Tse have shown that the bandwidth of a mobile ad-hoc network can be efficiently exploited adopting a simple 2-hop routing scheme, according to which data are transferred in the network through paths consisting of two hops: a data unit is sent from the source to a relay node, which physically carries the data around the network until it comes in contact with the destination node and delivers the data to it. It has been shown that the aggregate network throughput achieved by the 2-hop routing strategy increases linearly with the number of nodes $n$ in the network, in sharp contrast with previous results for networks composed of fixed nodes, where the network throughput increases at most with the square root of $n$ [2]. This nice property was first established in [1] under the assumption that nodes trajectories are independent, and result for each node into a uniform stationary distribution over the disk of unit area. In [3] the authors have considered an ad-hoc network in which each node independently moves along a randomly chosen great circle on the sphere of unit surface. Quite surprisingly, even under this one-dimensional mobility pattern the network throughput increases linearly with $n$ by adopting a simple 2-hop routing scheme. However, both network settings considered in [1], [3], which result in uniform contact times among the nodes, appear very idealistic. In real environments, contact times among the nodes are highly diverse, as recently pointed out in [4]. Indeed, an individual node usually spends most of the time just on a small portion of the entire network area, and rarely goes outside its region of habit. Therefore, we were motivated to consider, in this work, the performance of the 2-hop routing scheme in mobile ad hoc networks where node-to-node contact times are inhomogeneous.
We first summarize in Section II an analytical framework already introduced in [5] to formalize the definition of optimal network throughput in a mobile ad hoc network, in terms of a multi-commodity flow problem over the associated contact graph. Then we introduce in Section III several interesting properties relating the effectiveness of the 2-hop routing strategy to the structure of the contact graph. We also report experimental results which confirm and extend findings in [4] about the inhomogeneous nature of realistic contact graphs. At last, we show that in networks with inhomogeneous contact times the 2-hop routing strategy may result strongly inefficient. We conclude in Section IV.

\section{SYSTEM ASSUMPTIONS AND NOTATION}

We consider a mobile ad hoc network composed of $n$ nodes moving according to a general mobility model inside a bidimensional, (Lebesgue measurable) connected region $\mathcal{A}$ of area $|\mathcal{A}| . X_{i}(t)$ denotes the position of node $i$ at time $t$ and $\mathbf{X}(t)=$ $\left(X_{1}(t), X_{2}(t) \ldots X_{n}(t)\right)$ the vector of nodes positions; we define with $d_{i j}(t)$ the Euclidean distance between mobile $i$ and mobile $j$ at time $t$, i.e., $d_{i j}(t)=\left\|X_{i}(t)-X_{j}(t)\right\|_{2}$. We assume the node mobility process to be stationary and ergodic; i.e. given any $n$-uple $\left(B_{1}, B_{2}, B_{3} \ldots B_{n}\right)$ of Lebesgue measurable subsets of $A$, it results:

$$
\lim _{t \rightarrow \infty} \frac{1}{t} \int_{0}^{t} \mathbb{I}_{\left(\cap_{i} X_{i}(\tau) \in B_{i}\right)} d \tau=E\left[\mathbb{I}_{\left(\cap_{i} X_{i}(t) \in B_{i}\right)}\right] \quad \text { w.p.1 }
$$

where $\mathbb{I}$ represents the logical indicator function. Node $s$ generates traffic for destination $d$ according to a stationary and ergodic process with average traffic rate $\lambda_{s d} \mathrm{bit} / \mathrm{s}^{1}$. We denote with $\Lambda=\left[\lambda_{s d}\right]$ the corresponding $n \times n$ traffic matrix. We assume that interference between simultaneous transmissions is described by the well known protocol interference model [2]. According to the protocol interference model, transmission from node $i$ to node $j$ at time $t$ at rate $r$ is successful only if, for any other simultaneously transmitting node $k$, it holds:

$$
d_{k j}(t)>(1+\Delta) d_{i j}(t)
$$

for some guard factor $\Delta>0$. Note that according to this interference model: (i) no node can be either origin or destination of multiple simultaneous transmissions, (ii) a node cannot be simultaneously origin and destination of transmissions.

\footnotetext{
${ }^{1}$ Defined with $\hat{\lambda}(t, \tau)$ the amount of data generated by a source within the interval $[t, \tau)$, the traffic is said stationary and ergodic with average rate $\lambda$ iff: $E[\hat{\lambda}(t, t+1)]=\lambda$ for any $t>0$ and $\lim _{t \rightarrow \infty} \hat{\lambda}(0, t) / t=\lambda$ w.p. 1
} 
We denote with $E$ the set of all possible transmissionreceiver pairs $(i, j)$ (by construction it must be $i \neq j$ ). Subsets $\pi$ of $E$ in which nodes appear at most once (either as transmitter or receiver), represent possible transmission configurations, i.e., sets of transmission-receiver pairs $(i, j)$, which may be simultaneously enabled to communicate at time $t$. We denote with $\Pi$ the set of all the possible transmission configurations and with $A(t) \subseteq \Pi$ the set of all non-interfering (hence, implementable) transmission configurations at time $t$. The protocol interference model induces a map between the vector of instantaneous nodes positions $\mathbf{X}(t)$ and the set of non-interfering transmission configurations $A(t)$; we formalize this concept introducing function $\mathcal{I}$ mapping vectors of nodes positions into sets of non-interfering transmission configurations: $\mathcal{I}(\mathbf{X}(t))=A(t)$.

The scheduling policy $S$ dynamically selects an implementable transmission configuration $\pi^{S}(t)$ belonging to $A(t)=\mathcal{I}(\mathbf{X}(t))$. In this paper we restrict the investigation to the following stationary and ergodic scheduling policy, which was shown to be asymptotically optimal in [1], [3].

Definition 1: Given a network comprising $n$ nodes, policy $S^{0}$ schedules transmission between $i$ and node $j$ under the following condition:

$$
\min \left(d_{k j}(t), d_{k i}(t)\right)>(1+\Delta) d_{i j}(t) \quad \text { for } \Delta>0
$$

for every other node $k$ in the network (regardless of node $k$ activity).

Notice that, under scheduling policy $S^{0}$, the transmission bandwidth between $i$ and $j$ is equally shared in the two directions. Furthermore, according to scheduling policy $S^{0}$, a communication link is established between nodes $i$ and $j$ whose average capacity expressed in bit/s is:

$$
\begin{aligned}
\mu_{i j} & =\lim _{t \rightarrow \infty} \frac{1}{2 t} \int_{0}^{t} \mathbb{I}_{\left(\cap_{k \neq i, j} \min \left(d_{k j}(\tau), d_{k i}(\tau)\right)>(1+\Delta) d_{i j}(\tau)\right)} d \tau \\
& =\frac{1}{2} E\left[\mathbb{I}_{\left(\cap_{k \neq i, j} \min \left(d_{k j}(t), d_{k i}(t)\right)>(1+\Delta) d_{i j}(t)\right)}\right] \quad \text { w.p. } 1
\end{aligned}
$$

When movements of the nodes are independent, $\mu_{i j}$ can be obtained as function of the marginal spatial distributions of individual nodes:

$$
\begin{array}{r}
\mu_{i j}=\frac{1}{2} \int_{X_{i} \in A} \int_{X_{j} \in A}\left[\prod_{k \neq i, j} \int_{X \in A_{\Delta}\left(X_{i}, X_{j}\right)} d F_{k}(X)\right] \\
d F_{j}\left(X_{j}\right) d F_{i}\left(X_{i}\right)
\end{array}
$$

being $d F_{i}(X)$ the spatial Distribution of node $i$ and $A_{\Delta}\left(X_{i}, X_{j}\right)$ the area outside the transmission range of $i$ and $j: A_{\Delta}\left(X_{i}, X_{j}\right)=\left\{X: \min \left(\left\|X-X_{j}\right\|_{2},\left\|X-X_{i}\right\|_{2}\right)>(1+\right.$ $\left.\Delta)\left\|X_{i}-X_{j}\right\|_{2}\right\}$. A (capacitated) undirected graph $G(\mathcal{V}, \mathcal{E})$ whose vertices correspond to network nodes and capacitated edges correspond to communication links, univocally represents the mobile ad hoc network adopting $S^{0}$. In the following we refer to $G(\mathcal{V}, \mathcal{E})$ with the term contact graph. Therefore, the routing problem through the mobile ad hoc network adopting $S^{0}$ can be formalized in terms of a multi-commodity flow problem on the contact graph. Let $f_{i j}^{s d} \in[0,1]$ denote the average fraction of the traffic from node $s$ to node $d$, which is routed trough link $(i, j)$, i.e. for which $j$ follows $i$ as relay node [6]; $f_{i i}^{s d}=0$ by construction. The above quantities $f_{i j}^{s d}$ must satisfy the following well known flow conservation constraints:

$$
\sum_{i} f_{i j}^{s d}-\sum_{k} f_{j k}^{s d}=\left\{\begin{array}{cc}
1 & \text { for } j=d \\
0 & \text { for } j \neq d \text { and } j \neq s \\
-1 & \text { for } j=s
\end{array}\right.
$$

Proposition 1: A traffic matrix $\Lambda=\left[\lambda_{s d}\right]$ can be sustained iff the following multi-commodity flow problem defined by (2) and:

$$
\sum_{s d} \lambda_{s d} f_{i j}^{s d} \leq \mu_{i j} \quad \forall i, j
$$

admits a feasible solution. In such a case the set of variables $f_{i j}^{s d}$ univocally defines the routing strategy $R$.

An alternative, partial characterization of the sustainable region can be provided in terms of the capacities of contact graph cuts.

Proposition 2: Traffic $\Lambda=\left[\lambda_{s d}\right]$ is sustainable by policy $S$ only if, for any partition $(D, \bar{D})$ of the nodes, it results:

$$
\sum_{s \in \bar{D}} \sum_{d \in D} \lambda_{s d} \leq \sum_{s \in \bar{D}} \sum_{d \in D} \mu_{s d}
$$

It was proven in [7] that traffic $\Lambda$ is guaranteed to be sustainable if the ratio between the minimum value of the r.h.s and the maximum value of the 1.h.s is $\omega(\log f)$ being $f$ the number of flows. ${ }^{2}$ In case in which all traffic is originated at a single source and directed to a single destination (single commodity flow), the satisfaction of Proposition 2 is also sufficient for traffic sustainability, according to the well known max-flow min-cut Theorem. The set of all sustainable traffic matrices represents the capacity region of the mobile ad hoc network.

\section{PERFormance OF THE 2-HOP ROUTING STRATEGY}

In general, to efficiently exploit the network bandwidth, the routing strategy should minimize the average path length on the contact graph. This consideration justifies the fact that shortest path routing approaches have been widely employed in several application contexts related to computer communications [6]. In the context of mobile ad hoc networks, the 2-hop routing strategy has recently gained a wide popularity [1]; according to this strategy, data are delivered from source $s$ to destination $d$ either through a direct communication link, or through routes $s \rightarrow k \rightarrow d$, using every other node $k$ of the network as relay. Necessary and sufficient conditions for traffic to be sustainable under a 2-hop routing strategy are provided by the following statement:

Proposition 3: A traffic pattern $\Lambda=\left[\lambda_{s d}\right]$ is sustainable by the 2-hop routing scheme if the multi-commodity flow problem on the contact graph defined by (2) and (3) with the extra constraint: $f_{i j}^{s d}=0$ when both $i \neq s$ and $j \neq d$ admits a feasible solution.

${ }^{2}$ Given two functions $f(n) \geq 0$ and $g(n) \geq 0: f(n)=$ $o(g(n))$ means $\lim _{n \rightarrow \infty} f(n) / g(\bar{n})=0 ; f(n)=O(g(n))$ means $\limsup _{n \rightarrow \infty} f(n) / g(n)=c<\infty ; f(n)=\omega(g(n))$ is equivalent to $g(n)=o(f(n)) ; f(n)=\Omega(g(n))$ is equivalent to $g(n)=O(f(n))$ finally, $f(n)=\Theta(g(n))$ means $f(n)=O(g(n))$ and $g(n)=O(f(n))$. 
The set of all sustainable traffic matrices by the 2-hop routing strategy represents the 2-hop capacity region of the mobile ad hoc network. In the following we are interested to better understand the relationship between the capacity region and 2-hop capacity region on realistic contact graphs associated to mobile ad-hoc networks. To simplify the discussion, in analogy to what has been done in previous works [1], [2], [3], we assume that every node in the network is source and destination of a single information flow (i.e. $\Lambda$ contains only one non-null element per row and per column). In the following we refer to the above class of traffic patterns with the term "permutation traffic patterns". Of course, the performance of the 2-hop routing strategy can be arbitrarily bad on a general contact graph. For example, considering graphs with maximum nodal degree $d$, the average distance between nodes is $\Omega\left(\log _{d}(n)\right)$. Thus, for sufficiently large $n$, if traffic is exchanged only between node pairs whose distance on the contact graph is greater than two, the throughput sustained by the 2-hop strategy turns out to be zero. As we will see in the next section, contact graphs associated to mobile ad-hoc networks are typically strongly connected (i.e., $d=\Theta(n)$ ), and often fully connected (all edges exist). Nevertheless, we will show that, even in this case, 2-hop routing may result strongly inefficient, provided that capacities associated to the edges of the contact graph are highly unbalanced.

\section{A. Properties of the 2-hop routing strategy over general contact graphs}

Under a permutation traffic pattern, let us focus on a particular traffic relation $(s, d)$ and consider all paths from node $s$ to node $d$ followed by data according to a 2-hop routing strategy. The aggregate maximum transmission capacity obtainable by flow $s \rightarrow d$ on all parallel 1-hop and 2-hop paths of the graph is given by:

$$
\mu_{s d}^{2 h}=\mu_{s d}+\sum_{\substack{k \neq s, d \\ f \neq s}} \min \left(\mu_{s k}, \mu_{k d}\right)
$$

Moreover, the direct link from $s$ to $d$ is used exclusively by flow $(s, d)$. Link $(s, k)$ instead must be shared by flow $s \rightarrow d$ and the flow generated by $s^{\prime} \neq s, k$ and directed to $k$, whereas the capacity of link $(k, d)$ is shared by flow $s \rightarrow d$ and the flow generated by $k$ and destined to $d^{\prime} \neq k, d$. Hence, the capacity of each edge can be shared by at most two flows, due to the particular nature of the traffic matrix.

Sufficient conditions for traffic sustainability can be easily derived under the 2-hop routing strategy, assuming that bandwidth of links is evenly partitioned among the flows.

Proposition 4: Under the 2-hop routing strategy, a permutation traffic pattern is guaranteed to be sustainable if for every source and destination pair $(s, d)$ the following constraint is met:

$$
\lambda_{s d} \leq \mu_{s d}+\frac{1}{2} \sum_{k \neq s d} \min \left(\mu_{s k}, \mu_{k d}\right)
$$

It is interesting to understand under which conditions the 2-hop routing strategy is guaranteed to efficiently exploit the system bandwidth. The following theorem provides an answer to this question.

Theorem 1: If for every pairs of nodes $(s, d), \mu_{s d}^{2 h}$ equals the minimum cut capacity between $(s, d)$, then the 2-hop routing scheme is $1 / 2$-efficient in terms of throughput, i.e., if $\Lambda=\left[\lambda_{s d}\right]$ can not be sustained by the 2-hop routing strategy, no other routing strategy can sustain traffic $2 \Lambda=\left[2 \lambda_{s d}\right]$.

We remind that, given any partition of graph vertices $(S, D)$ with $s \in S$ and $d \in D$, the capacity $\mu_{(S, D)}$ associated to the cut $(S, D)$ is defined as: $\mu_{(S, D)}=\sum_{i \in S} \sum_{j \in D} \mu_{i j}$. The mincut capacity $\hat{\mu}_{s d}$ between $(s, d)$ is defined as $\min _{(S, D)} \mu_{(S, D)}$.

Proof: Consider any source destination pair $(s, d)$. Under a general routing strategy the amount of traffic $\lambda_{s d}$ that can be sustained by the network can not exceed the min-cut between $s$ and $d$ on the contact graph. On the other side the 2-hop routing strategy sustains between $(s, d)$ a traffic $\lambda_{s d} \geq \frac{1}{2} \mu_{s d}^{2 h}$ according to Proposition 4 . The assert follows immediately, since $\mu_{s d}^{2 h}=\hat{\mu}_{s d}$ for all $s$ and $d$.

Since $\sum_{k} \mu_{s k}$ and $\sum_{k} \mu_{k d}$ are two cuts, the condition:

$$
\mu_{s d}^{2 h}=\min \left(\sum_{k \neq s, d} \mu_{s k}, \sum_{k \neq s, d} \mu_{k d}\right)
$$

is sufficient to guarantee that at least half of the optimal throughput is delivered by the 2-hop routing strategy, under any permutation traffic pattern.

Note that, when nodes move uniformly over the network area $\mathcal{A}$, by symmetry all link capacities $\mu_{i j}$ are equal, thus $\mu_{s d}^{2 h}=\hat{\mu}_{s d}$ for all $s$ and $d$. Furthermore in this specific case the 2-hop strategy can be easily shown to be 1-efficient.

For a general contact graph $G(\mathcal{V}, \mathcal{E})$, define

$$
\eta^{2 h}=\min _{s d} \frac{\mu_{s d}^{2 h}}{\hat{\mu}_{s d}}
$$

The statement of Theorem 1 can be generalized to the case $\eta^{2 h}<1$ :

Theorem 2: Provided that $\eta^{2 h}=\alpha<1$, the 2-hop scheme is $\alpha / 2$-efficient in terms of throughput, i.e., if $\Lambda=\left[\lambda_{s d}\right]$ can not be sustained by the 2-hop scheme, no other routing strategy can sustain traffic $\frac{2}{\alpha} \Lambda=\left[\frac{2}{\alpha} \lambda_{s d}\right]$.

Furthermore there exists a permutation traffic pattern $\Lambda$ which is sustainable by a properly defined routing scheme and such that $(\alpha+\epsilon) \Lambda$, for any $\epsilon>0$, is not sustainable by the 2-hop strategy.

Proof: The first statement can be proved using exactly the same arguments of Theorem 1.

For what concerns the second statement, consider the source-destination pair $(s, d)$ in correspondence of which $\mu_{s d}^{2 h} / \hat{\mu}_{s d}=\alpha$. If only $(s, d)$ exchanges traffic in the network, the maximum amount of traffic that can be sustained in the network by an arbitrary routing strategy equals the min-cut $\hat{\mu}_{s d}$; on the other hand according to the 2-hop scheme the maximum amount of traffic that can be sustained can not exceed $\mu_{s d}^{2 h}$.

In conclusion, if $\eta^{2 h}=\alpha$ we should be prepared to get a throughput reduction of order $1 / \alpha$ in case the 2-hop routing strategy is adopted.

\section{B. Experimental mobile networks}

We have analyzed two experimental mobile ad hoc networks and derived the corresponding contact graphs: 


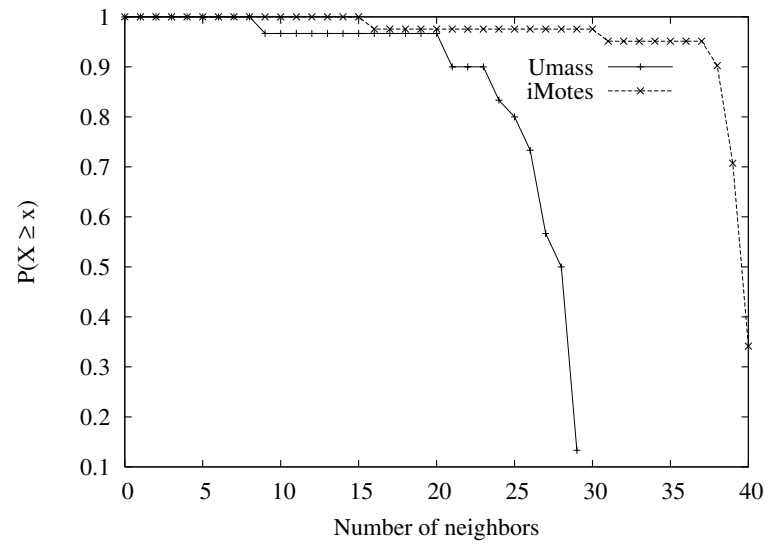

Fig. 1. Tail distribution functions of the number of neighbors of a node

- Umass network: data are obtained from DieselNet [8] network at Umass campus during the Spring quarter in 2005. This network consisted of 30 buses running the campus transport service and carrying some short range radio devices. The publicly available traces [8] refer to the radio contacts among buses, measured in terms of data transferred through TCP connections. We considered the capacity among the nodes (buses) in terms of aggregate amount of data (Mbytes) transferred during the measurement interval (about 60 days).

- iMotes network: data are obtained from the iMotes experiment that took place during Infocom 2005 conference [4]. A set of 41 small intelligent network devices (implemented through iMotes) were carried in the pocket by some volunteers attending the conference; the iMotes had small radio range and, thanks to the mobility of the person, they could contact other iMotes. The publicly available traces of the experiment [4] provide the radio contact durations (i.e., the capacity measured in seconds) between any two iMotes nodes ${ }^{3}$.

The two networks are derived from two different mobility patterns: buses of Umass followed almost predetermined paths, whereas iMotes nodes moved in an unpredictable way.

Both contact graphs are almost fully connected, since about $86 \%$ (Umass) and 95\% (iMotes) of all the possible edges exist; each node is connected to almost all the other nodes, as Fig. 1 shows. It is interesting to note, as already observed in [4], that the distribution of the edge capacities in iMotes appears as heavy-tailed; Fig. 2 shows the tail distribution function of the edge capacities for both networks. The contact graphs of the two networks contain significant inhomogeneous capacities; Fig. 3 shows visually the contact graphs partitioned in three subgraphs: the left subgraphs contain edges with small capacity (class 1 edges), the central subgraphs (almost fully connected) contain medium capacity edges (class 2 edges), whereas high capacity edges are shown in the right subgraphs

\footnotetext{
${ }^{3}$ The traces refer also to some external devices, but since the information on their direct contacts is not available, we restricted our analysis to iMotes devices only.
}

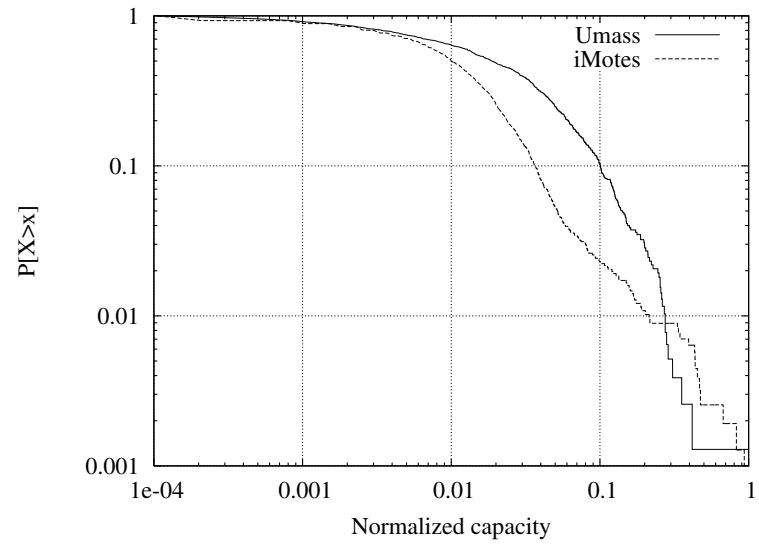

Fig. 2. Tail distribution functions of the edge normalized capacities

(class 3), in which we have also highlighted in bold all of the edges with very high capacity (class 4 ). For coherence, we considered the normalized capacity of an edge, defined as the ratio between the edge capacity and the maximum capacity available in the corresponding network. Observe that, even if a small fraction of the overall edges have high capacity, they contribute to most of the overall transport capability: this is especially true for iMotes.

While maximizing $\sum_{s d} \lambda_{s d}$, through the solution of the multi-commodity flow problem described in Sec. II, we have evaluated the maximum achievable throughput for two specific permutation traffic scenarios, assuming that all nodes generate the same amount of traffic. The 30 (Umass) and 41 (iMotes) node pairs that exchange traffic in the two scenarios (named minimum Direct Capacity - $m D C$ - and Maximum Direct Capacity - $M D C$, respectively), have been selected so as to minimize/maximize the sum of capacities associated to the direct communication links between sources and destinations.

Table I shows the aggregate maximum throughput, the average and the maximum number of hops for the corresponding optimal routing strategy. Under optimal, unconstrained routing, the maximum number of hops is always equal to 5 ; Fig. 4 shows the distribution of the number of hops for both networks; under $m D C$ the number of direct paths is minimized. We also show the maximum throughput obtained by adding the constraints in the multi-commodity flow problem that only 1 and 2 hops routes are available: $f_{i j}^{s d}=0$ if both $i \neq s$ and $j \neq d$. From Table I it is also possible to evaluate the loss of throughput due to 2-hop routing with respect to the optimal unconstrained routing: 21\% (Umass) and 30\% (iMotes) loss for $m D C ; 16 \%$ (Umass) and $10 \%$ loss (iMotes) for $M D C$. The loss of throughput is higher for $m D C$ scenario, where the traffic flows employ longer paths.

At last, in Fig. 5, the values of $\eta_{s d}^{2 h}=\mu_{s d}^{2 h} / \hat{\mu}_{s d}$ for all source-destination pairs are displayed in increasing order.

The average values of $\eta_{s d}^{2 h}$ for Umass and iMotes network are 0.603 and 0.535 respectively, suggesting that moderate throughput degradations are experienced employing a 2-hop routing strategy under most traffic scenarios, like the ones pre- 
Umass - Class 1

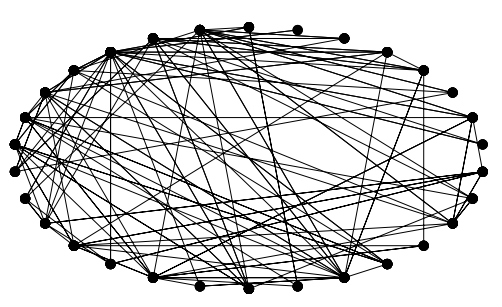

iMotes - Class 1

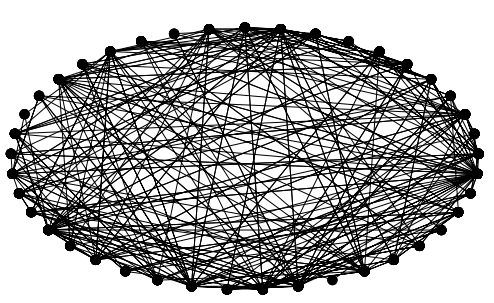

Umass - Class 2

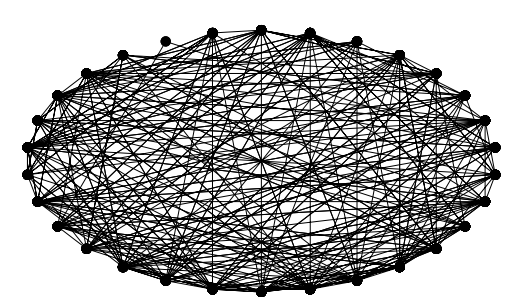

iMotes - Class 2

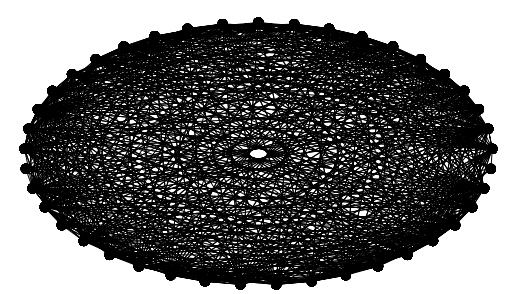

Umass - Class 3-4

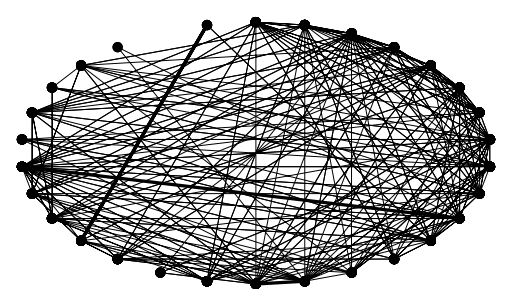

iMotes - Class 3-4

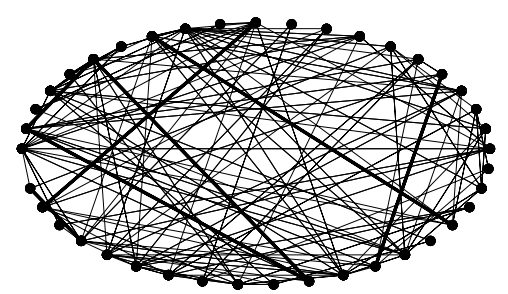

Fig. 3. Contact graph of Infocom-iMotes experiment, subdivided in three subgraphs: class 1 edges with normalized capacity $<0.003$, class 2 edges with norm. cap. $0.003-0.03$, class 3 edges with norm. cap. $0.03-0.3$, class 4 edges (in bold) with norm. cap. $>0.3$. Classes 3-4 contribute for $86 \%$ (Umass) and $60 \%$ (iMotes) of the overall transport capacity and for $40 \%$ (Umass) and $14 \%$ (iMotes) of the total number of edges.

\begin{tabular}{|c|c|c|c|}
\hline $\begin{array}{c}\text { Traffic } \\
\text { scenario }\end{array}$ & $\begin{array}{c}\text { Maximum aggregate } \\
\text { capacity }\end{array}$ & $\begin{array}{c}\text { Average } \\
\text { num. hops }\end{array}$ & $\begin{array}{c}\text { Max } \\
\text { num. hops }\end{array}$ \\
\hline \multicolumn{4}{|c|}{ Unconstrained optimal routing } \\
\hline Umass $m D C$ & 21,131 Mbytes & 2.31 & 5 \\
Umass $M D C$ & 28,578 Mbytes & 1.85 & 4 \\
\hline iMotes $m D C$ & $1.75610^{6} \mathrm{sec}$ & 2.60 & 5 \\
iMotes $M D C$ & $3.00810^{6} \mathrm{sec}$ & 1.69 & 5 \\
\hline \multicolumn{4}{|c|}{$1-2$ hops optimal routing } \\
\hline Umass $m D C$ & $16,622 \mathrm{Mbytes}$ & 1.96 & 2 \\
Umass $M D C$ & $24,045 \mathrm{Mbytes}$ & 1.58 & 2 \\
\hline iMotes $m D C$ & $1.22810^{6} \mathrm{sec}$ & 1.99 & 2 \\
iMotes $M D C$ & $2.72010^{6} \mathrm{sec}$ & 1.50 & 2 \\
\hline
\end{tabular}

TABLE I

OPTIMAL CAPACITY THROUGHOUT OPTIMAL ROUTING FOR UMASS-BUSES AND INFOCOM-IMOTES EXPERIMENTS

viously selected. Nevertheless, the values of $\eta^{2 h}=\min _{s d} \eta_{s d}^{2 h}$ are rather small in both cases: $\eta^{2 h}=0.1234$ for the Umass network and $\eta^{2 h}=0.0180$ for the iMotes network. As a consequence significant throughput degradations are experienced for some worst-case traffic scenarios, according to which traffic is exchanged only by node pairs having small $\eta_{s d}^{2 h}$.

\section{Asymptotic throughput loss of the 2-hop routing strategy}

In this section we focus on fully connected contact graphs with inhomogeneous capacities, such as those emerging from experimental setups. We assume that edge capacities $\mu_{i j}$ are i.i.d. random variables with assigned distribution. In this case we are able to show analytically that, for $n \rightarrow \infty$, the 2-hop routing strategy can be strongly inefficient, while the throughput loss depends on the particular edge capacity

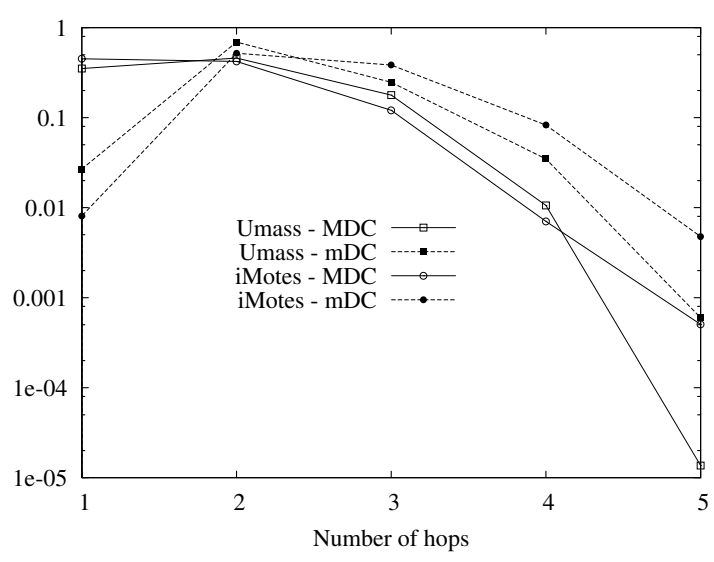

Fig. 4. Distribution of the number of hops under throughput optimal (unconstrained) routing

distribution. Essentially, we need to evaluate parameter $\eta^{2 h}$ introduced in (6). Due to the symmetry of the contact graph, we consider a randomly chosen source-destination pair $(s, d)$ and estimate the ratio between the aggregate capacity of 2-hop paths and the minimum capacity among all cuts between $s$ and $d$, as $n$ grows large. In Fig. 6 the contact graph is represented for $n=6$. As $n$ increases, to keep finite the capacities of the cuts to be evaluated, edge capacities are scaled by $n$, i.e., their distribution is given by $f_{\mu}^{n}(x)=n g(n x)$, being $g(x)$ an assigned distribution function. As a consequence, the average capacity of each edge is $E\left[\mu_{i j}\right]=\frac{\mu}{n}$, where $\mu=\int x g(x) d x$. Note that the scaling effect of edge capacities is intrinsically obtained when contact capacities are computed 


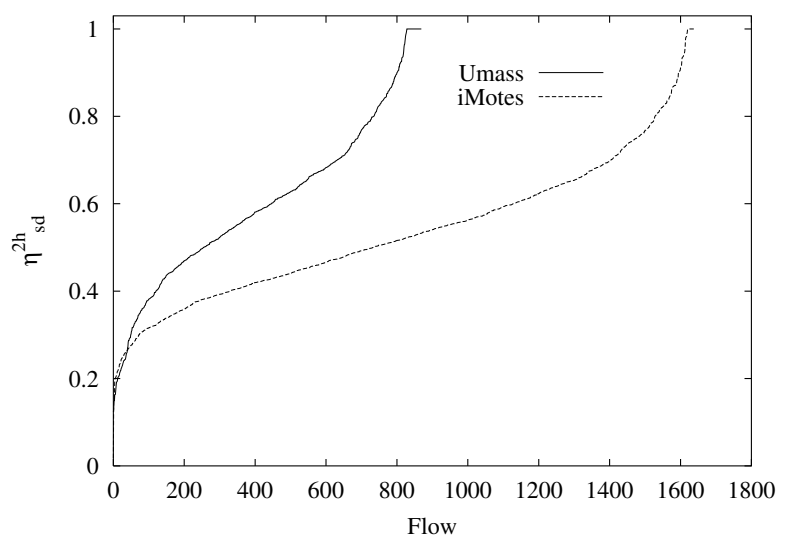

Fig. 5. Values of $\eta_{s d}^{2 h}=\mu_{s d}^{2 h} / \hat{\mu}_{s d}$ sorted in increasing order are plotted for for source destination pairs

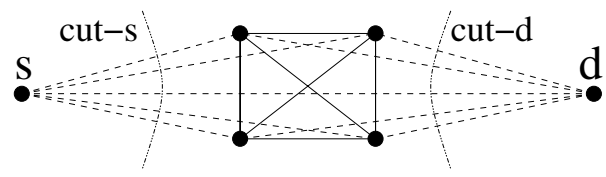

Fig. 6. Contact graph for $n=6$.

according to (1) (see [1], [5]). Given two independent random variables $X$ and $Y$ distributed according to $g(x)$, we denote with $\mu_{\min }=E[\min (X, Y)]$.

Observe that the cut corresponding to node partition $(S, D)$ with $|S|=k$ and $D=n-k$, being $k=1, \ldots, n-1$, contains $k(n-k)$ edges; thus the cut around the source ("cut-s") (i.e, the cut for which $S=\{s\}$ ) and the cut around the destination ("cut-d") (i.e., $D=\{d\}$ ), are minimal in terms of number of edges traversing them $(n-1)$. It can be shown (we omit here the details of the proof due to lack of space) that, for $n$ large enough, with high probability (i.e., with probability that tends to 1 when $n \rightarrow \infty$ ) the minimum-capacity cut corresponds to either "cut-s" or "cut-d".

The maximum achievable flow from $s$ to $d$ corresponds to the minimum between cut-s and cut-d:

$$
\hat{\lambda}_{s d}=\min \left(\sum_{k \neq s} \mu_{s k}, \sum_{k \neq d} \mu_{k d}\right) \rightarrow \mu \quad \text { w.p.1 }
$$

when $n \rightarrow \infty$. If we allow only 2 -hop routing, the maximum achievable throughput $\lambda_{s d}^{2 h}$ is given by the aggregate capacity on all $n-1$ parallel 1-hop and 2-hop paths between $s$ and $d$ :

$$
\lambda_{s d}^{2 h}=\mu_{s d}+\sum_{k \neq s, d} \min \left\{\mu_{s k}, \mu_{k d}\right\} \rightarrow \mu_{\min } \quad \text { w.p.1 }
$$

when $n \rightarrow \infty$. Hence, asymptotically for $n \rightarrow \infty, \eta^{2 h}$ is given by:

$$
\eta^{2 h}=\min _{s d} \frac{\lambda_{s d}^{2 h}}{\hat{\lambda}_{s d}} \leq \frac{\mu_{\min }}{\mu}
$$

We notice that, under the condition that the fourth moment of $g(x)$ is finite, the above upper bound on $\eta^{2 h}$ can be easily proved to be tight as $n \rightarrow \infty$.

It is interesting to compute $\mu_{\min } / \mu$ for specific distributions of edge capacities. We consider two cases, both simple to evaluate analytically:
- $g(x)$ is an exponential distribution with mean $a$ (i.e., $g(x)=e^{-x / a} / a$, for $\left.x \geq 0\right)$ then $\min \left\{\mu_{s k}, \mu_{k d}\right\}$ is still exponential with mean $a / 2: \eta^{2 h} \leq 1 / 2$.

- $g(x)$ is a Pareto distribution with parameters $a$ and $b$ (i.e., $g(x)=b a^{b} / x^{b+1}$, for $x \geq a$ and $b>1$ ), having mean $a b /(b-1)$; then $\min \left\{\mu_{s k}, \mu_{k d}\right\}$ is still Pareto with parameters $a$ and $2 b: \eta^{2 h} \leq 2(b-1) /(2 b-1)$. Hence, the bound on $\eta^{2 h}$ is an increasing function starting from 0 for $b \rightarrow 1$ and growing to 1 only for $b \rightarrow \infty$. Note that $b \in(1,2]$ corresponds to capacities with finite mean but infinite variance.

Hence, for exponentially distributed capacities, under a generic permutation traffic pattern we can expect 2-hop routing to be slightly inefficient, leading to throughput loss within a factor 4 (by Theorem 2). For Pareto-distributed capacities, instead, 2-hop routing can be very inefficient leading to unbounded throughput loss when $b \rightarrow 1$.

In conclusion, in case of inhomogeneous edge capacities, paths of several hops are needed to efficiently exploit the system bandwidth, also when the associated contact graph is fully connected.

\section{Conclusions}

In this paper we have considered mobile ad hoc networks under realistic node mobility patterns, and we have studied the throughput performance of the 2-hop routing scheme. We have related the effectiveness of the 2-hop routing strategy to the structural properties of the contact graph associated with the network. We have then presented experimental evidence that contact times between nodes are highly inhomogeneous in a real environment. At last we have shown that, in realistic networks, the 2-hop routing strategy may result strongly inefficient in terms of aggregate throughput.

\section{REFERENCES}

[1] M. Grossglauser, D.N.C. Tse, "Mobility increases the capacity of ad hoc wireless networks", IEEE Trans. on Networking, Vol. 10, n.2 pp. 477-486

[2] P. Gupta, P.R. Kumar, "The capacity of wireless networks", IEEE Trans. on Information Theory, Vol. 46, n.2, pp. 388-404

[3] S.N. Diggavi, M Grossglauser, D.N.C. Tse, "Even one-dimensional mobility increases ad hoc wireless capacity", IEEE Trans. on Information Theory, Vol. 51, n. 11, pp. 3947-3954

[4] P. Hui, A. Chaintreau, J. Scott, R. Gass, J. Crowcroft, C. Diot, "Pocket Switched Networks and the Consequence of Human Mobility in Conference Environments", ACM WDTN, Philadelphia, PA, August 2005

[5] M.Garetto, P.Giaccone, E.Leonardi, "On the Capacity of Ad Hoc Wireless Networks Under General Node Mobility", Techical report, available at http://www.tlc-networks.polito.it/database

[6] D. Bertsekas, R. Gallager, Data networks, Prentice-Hall, 1992.

[7] Y. Aumann and Y. Rabani, "An $O(\log k)$ Approximate Min-Cut MaxFlow Theorem and Approximation Algorithm", SIAM Journal on Computing, Vol. 27, n. 1, 1998.

[8] John Burgess, Brian Gallagher, David Jensen, Brian Neil Levine, "MaxProp: Routing for Vehicle-Based Disruption-Tolerant Networking," in Proc. IEEE Infocom 2006, Barcelona, Spain, April 2006. 